\title{
Limited Animation as A Means of Spreading Individual Thought Through Social Media
}

\section{Dr. Maha Mansour Mohamed Ali Soliman}

\section{Abu Qir Officers' Residence, Building 27C, Apartment 41, Alexandria, Egypt}

\section{Abstract:}

The social media system is part of the fabric of modern society or the so - called "society media" and during a short period of time it has become central to the free dissemination and self - expression of the user, prompting governments to allocate services within ministries to follow modern mass media.

Like traditional media, this social communication has had its share of guidance and the formation of attitudes and trends, but the difference in that the source of intellectual guidance here comes not from a State, organization or policy of a media channel, as in traditional media, but from the different individual platforms in thought, orientation and direction that seek to attract more followers, and the power of attraction and follow-up depends on the ability of the individual to communicate his or her own intellectual content attractively. The expression process consisted of multiple forms, including limited animation.

Key words: Limited Animation - Society media - Citizen Media - Dependence theory - Uses theory and needs satisfaction - Virtual community - Virtual Citizenship.

\section{Introduction:}

Ideas in human life are messages that are transformed and mobile between time and space. Thought is a message that does not dissolve its owner, but that travels from one person to another in different ways and different ways, according to each time and place. It is embraced by one person, then taken to be transformed by another, and then a third comes to be built on by multiple other ideas.

The dissemination of thought is a color of expression, a message that is shaped and able to move from one person to another. These messages are in a media space limited by time. From print newspapers and to social media, a significant time gap is the link between traditional journalism and citizen journalism, or traditional and popular media.

During this period, the form of these expressed messages is many. They are spoken, written, painted or animated, in proportion to the concept of the means of information used and the limitations they define. These concepts are reflected in the electronic media theories of interdependence, use theory and satisfaction of needs, which are the key to interpreting the 
promotion of individual thought through the use of limited animation, and thus contribute to shaping public opinion:

- What's the effectiveness of limited animation in spreading individual thought and mobilizing public opinion?

- What's the connection between limited animation features and the social media concept?

- What are the reasons for the limited social media cartoons from media theories?

\subsection{Individual intellectual exchange in virtual community:}

The Arab world has known many events in the modern era. As usual, large-scale events have a cultural and social reaction. As a result of constraints in societies in general and Arab societies in particular, virtual community have emerged in the form of a cyber space in which robotic human interaction occurs mentally, psychologically and socially in various human senses as well as mechanically .In this space as well, the virtual community is composed of individuals and groups in an automated environment, so that communication is in the various spheres, fields and activities available to man. Virtual community is a group of human beings with common interests and not necessarily with geographical boundaries or ethnic, tribal, political or religious ties .They interact through modern media and social media, and develop among themselves the conditions of community membership, entry and exit rules, coping mechanisms, rules and ethics that should be observed, clearly through social media (Basma Kqed Albanaa, 2014, p134).

Social media has emerged as a set of web applications built using second-generation web technology, which allows users to create and share automated content, that is, applications that enable users to create pages - personal files - that connect them to their friends and acquaintances and enable them to communicate with others by sharing personal messages via the email of the user's profile. Or by leaving the various comments that users share, those personal files or pages may contain images, video files or audio files .The social media term "Social Media" is divided into two parts: Part I "Media" means any media that allows individuals to communicate together, including traditional means such as radio, television and newspapers, while "Social" means that the process is a two-way process from the sender to the 
receiver (message) and at the same moment from the receiver to the sender (reaction) (Nermin Alaa Elden, 2020, p11).

Social media sites have emerged, and the number of users has increased at different ages and cultures. They have become one of the most important sources through which people have access to information. They communicate with individuals, exchange ideas, mobilize and shape public opinion. The individual became a recipient and sender at the same time, appearing with so-called popular media, or so-called citizen press, where the individual used himself became the source of information .Major social media sites include Facebook, Twitter, YouTube, LinkedIn, Instagram .

\subsubsection{Citizenship and virtual freedom of expression:}

The existence of social media has added another phenomenon, the so-called virtual citizenship. In the light of the tension experienced by States and the lack of rights and freedoms in societies, a virtual society has become the window through which an individual can express his or her opinion and spread his or her personal thoughts on the social issues and problems he or she faces, without any control that limits his or her ability to express (Basma Kqed Albanaa, 2014, p106).

The term "citizen's press" has emerged from this concept. Instead of limiting expression to the intellectual public, expression has become the right of the ordinary citizen or people. A citizen must move from a mere future and a consumer to a conscious participant and actor responsible for his or her educational and media behavior in the context of dependency, tradition and drift, where he or she must find the alternative .In a press that is responsible, committed and conscious of the issues of the vast majority of the people and of sensitive, serious and critical issues, to present, review and discuss in order to involve all to contribute and propose successful solutions to address them.

Freedom of expression is the result of freedom of belief, that is, freedom of thought and belief in what we believe to be the truth. Freedom of expression is an important right of citizens in modern societies .Freedom of expression is one of the most fundamental freedoms for the human person, as the human person has no value when he loses the freedom to express his opinion. Freedom of expression is therefore essential for the realization of the identity of 
individuals, the circulation of ideas and opinions and the establishment of the principles of democracy and citizenship in all societies (Khaled Mohamed Gazi, 2010, p198, 246).

\subsubsection{Citizen Media:}

It is a new phase of journalism and media production, a participatory press emanating from blogging, which is a form of self-realization, an intellectual activity that brings one's victory over linear models of communication, and citizen journalism synonymous with popular media. However, the term citizen journalism has emerged in the West to describe the media practices of communities far from major cities, which have targeted the provision of news coverage to themselves in the absence of state coverage by major media .The term citizen appeared in the West because citizenship was the umbrella that could cover everyone. Some felt that citizen journalism was incompatible with the general culture of the Arab region, because the word citizen had not been in circulation for some time under the domination of authoritarian regimes. "People" were most common among Arabs, especially since the combination gave a more solidarity meaning than the individual citizen (Basma Kqed Albanaa, 2014, p56, 57, 86), (Noha Atef, 2016, p27, 28).

\subsubsection{Society Media:}

It is a modern term that has emerged as a result of the emergence of the new media. It is an information made by the people from text, audio or visual material. It is disseminated through the network media by any individual outside the governance institution. The essence of the popular media lies in erasing the line between the public and journalists .If a journalist provides content, everyone becomes an audience for as long as it is consumed by reading or watching .Popular media differs from traditional media in several points:

- The administrative structure is missing and replaced only by an individual.

- The competitive environment among popular journalists has declined considerably.

- The absence of quality standards in popular media and reliance on experimentation.

- Failure to adhere to good journalism standards.

- Moving away from objectivity in building content, where the journalist is part of the event (Noha Atef, 2016, p23). 


\subsubsection{Mobilizing virtual public opinion :}

Virtual public opinion is any idea, proposal, opinion, participation or expression of an angry objection or joke that expresses a particular orientation, defends a particular ideology, or proceeds from a personal experience, whether individual or collective, to reach a general political conclusion delivered as a communication message through social media to be seen and seen by anyone who owns or can use that service .In this case, virtual public opinion expresses all the segments that possess that mean or technological tool of expression, communication and debate.

The process of mobilizing public opinion is the process of consolidating the visions and trends behind an issue, which is actually making a radical difference. The process of mobilizing on social media by drawing users automatically and spontaneously, in contrast to the systematic and funded polarization that exists in the traditional media.

The mobilization of public opinion is linked to two studies linked to the concept of gatekeeper, an entity that has the discretion to exercise gatekeeping through the network guard mechanism, divided into:

Rallying public opinion: Public opinion is directed by gatekeepers by passing the selected contents to public opinion, whether misleading or false, through:

- Pass on contents consistent with the approach adopted by the Authority.

- Passing on content with high potential in terms of its media output.

- Pass two contents that are relevant to the gatekeeper and achieve a certain benefit.

Crowd of unsuspecting public opinion: It's the opposite of a structured crowd. He has the advantage of being more credible, aware and enlightening because:

- The credibility of social networks stemming from the fact that the citizen is the media, as the user sees his friends, relatives and neighbors trading news, the user trusts the content according to his confidence in the content product. 
- These networks provide a collective space of different ideologues and opinions, left, right, and middle, with their virtual voice, and the networks are therefore not biased towards one stream at the expense of the other.

- Mobilization and polarization are done spontaneously

\subsubsection{The Concept of Challenge the Silence:}

New media and social networks are a source of information, disclosure of issues, absentee information and expression of views and views ‘.The more these sites are used among members of society, the higher the levels of participation and participation, and the wider the expression of public opinion ، As a result, the phenomenon of belief in the dominance of the mainstream opinion resulting from the adoption of traditional media and the phenomenon of strengthening silence and fear of isolation have disappeared. This can be formulated through the role played by media sites, communication tools and interaction among people in challenging silence (Mohamed Mostafa Refaat, 2018, p90, 91, 67).

\subsubsection{Media Fragmentation}

The concept of mass fragmentation or the so-called fragmented audience has emerged as a result of the multiplicity of modern means of communication, the diversity and wealth of information and media production. New media audiences are no longer the big, unified and homogeneous masses themselves, as they are in traditional media, but have become small units . Everyone receives from his or her own outlet various items that suit him or her, increasing the choices of media consumers, and their time is spread between websites, social networks, smartphones and video games, as well as other traditional media .As a result of this mass fragmentation, new features have emerged:

- Diversity and coverage of digital mobile content.

- Multiculturalism and different trends between the parties to the process.

- achieving proliferation, universal access and the ability to transcend geographical boundaries.

- freedom, accessibility and access to information.

- Expanding media competition.

- The growing role of the private sector in media and advertising work. 
- The emergence of a new form of media called individual media.

\subsubsection{The Origin of Individual Information (Dissemination of Individual Thought):}

The information of the individual has emerged as a new form of information, as a result of the fragmentation of the masses. It has become the focus of personal broadcasting and media content production, through modern technologies, such as video cameras, design programs, various applications, writing on sites and blogs, and its dissemination through various social media sites. Most notable among these are the special YouTube channels and the programs and episodes produced and produced by people, some of whom do not belong, they address topics that touch on community-related issues and raise citizens' concerns, which are highly watched content and receive high echoes and widespread public welcome (Basma Kqed Albanaa, 2014, $\mathrm{p} 32,33)$.

\subsection{Individual expression in society media}

The dissemination of individual thought on social media in the new media has appeared on various bodies and images, and these bodies have helped the individual to express his or her personal thoughts or to express other ideas that the Authority prevents from publishing, the expression came in the form of:

- Expression through literary text: In the form of literary or slang text publications, the individual used to publish his or her ideas in proportion to everyone, not only writing in proper grammar, but also in the street vernacular.

- Expression through images and drawings: It appeared through images or drawings posted through users' personal pages and public pages.

- Expression through motion pictures (video): has emerged through user use of phones and cameras to film scenes that are shared by an individual or issues affecting his or her personal interest. 
- Expression through Limited Animation: This kind of animation has appeared parallel to street language literature. Just as a citizen uses street language or vernacular language to express himself, he has also resorted to limited animation for the purpose of expression and in a simplistic way mixes spontaneity with good communicative effectiveness. (Full animation, which is highly professional, but animated, with the intellectual content easily accessible to the street man .

\subsubsection{Individual expression in society media via limited animation:}

Limited animation is a form of abstract animation in which movement is limited to the necessary frames, and abstraction refers not only to the execution method of the graphics themselves, but also to the way in which the graphics move. Some abstract graphics have been fully animated, although they exist in an abstract way in the drawing. On the contrary, some of the animations are multi-detail and the parts are finely animated in limited way.

And limited animation is shown for a long time on screen like a cartoon, so the artist plans it carefully, taking into account the economy in the production side ، .So the iteration of some drawings is used whenever possible within a second, and this method is used to reduce the number of drawings made by a painter and rely only on twelve, eight or six variable drawings within a second instead of 24 frames per second ‘.Hence, these limited graphics require a high degree of execution skill, such as complete animation. The artist here has to create an illusion of action and reaction with a few expressions (Harold Whitaker, John Halas, 2009, p1).

\subsubsection{Techniques in limited animation}

Limited animation does not reduce the cost of working at the expense of the creative side of the work, Limited animation relies on different methods of showing motion while maintaining the expressive aspect of the work, such as:

The Staccato system: It's an Italian term that belongs to the musical sound-shredding characteristics, which are similar to the intermittent animation style, which is different from full animation using 24 frames, 12 frames or eight frames per second.When the character's face is moved, only two frames are set, one front and the other side of the face, and when they move quickly, it appears as if the character is moving its head. In this way, it is similar to the work of 
the artist Pablo Picasso and the artist Mattis, where the presence of low-number tires and the simultaneous entanglement of static images make the effect of the syntactic graphics

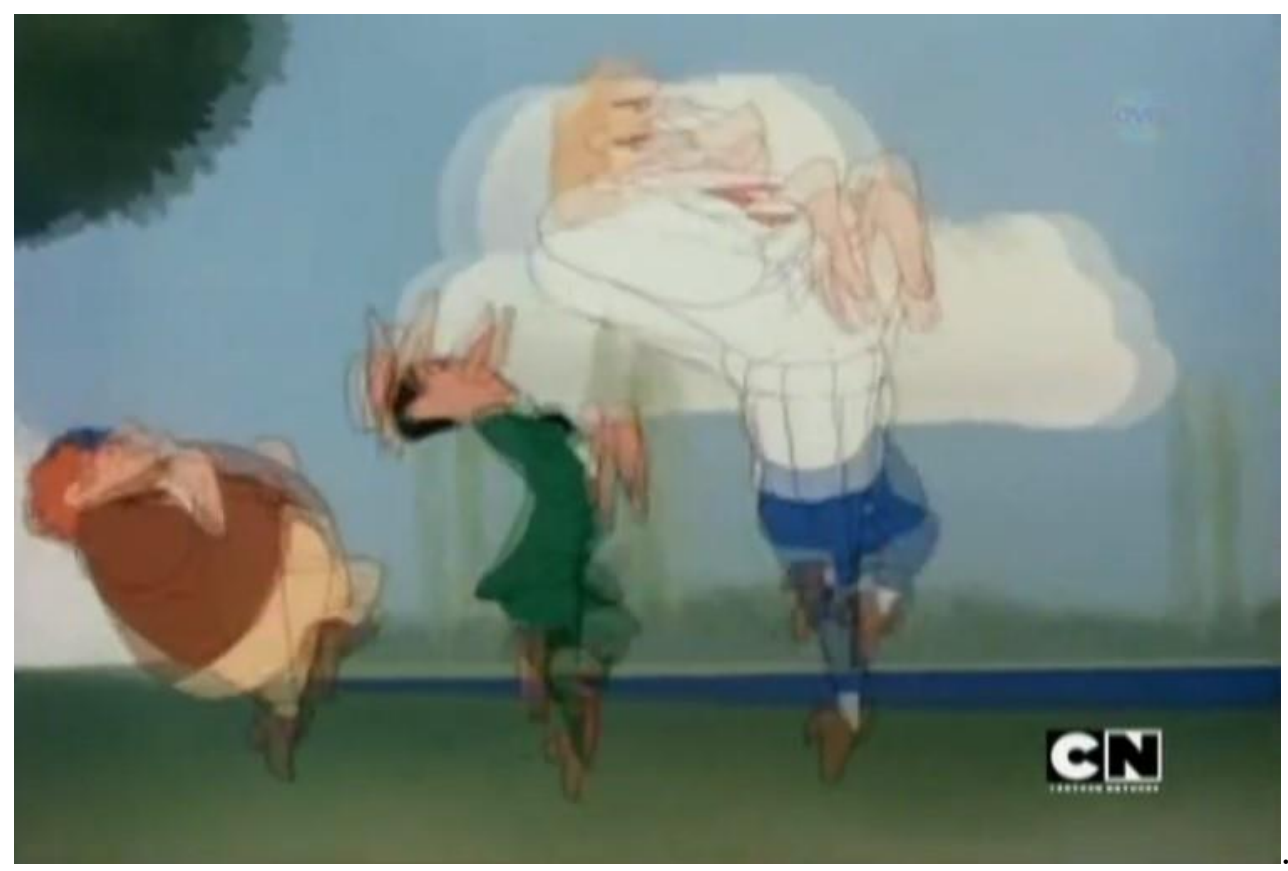

Fig. 1. the staccato system- The Stretch system The Dover Boys at Pimento University or The Rivals of the Roquefort Hall- 1942

The Stretch system: This technique has been used in some Disney animated films because it has a comic effect appropriate to the comic content of these cartoons.
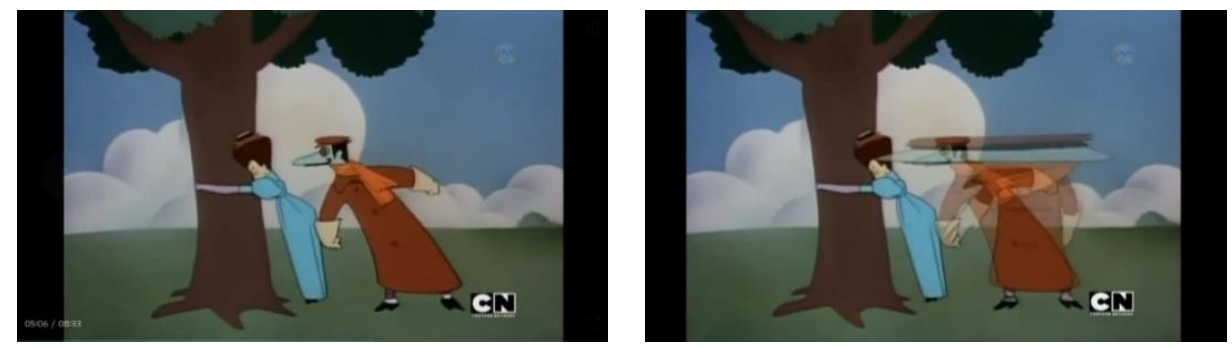

Fig.2. The Stretch system -The Dover Boys at Pimento University or The Rivals of the Roquefort Hall- 1942 
The slash system: This technique came out of time, as artists used it earlier in the silent animation period to make animation easier ، This method has come to solve the problem of replicating the graphics completely during the character movement, where in this system the scene is drawn once, leaving an empty space for the moving part .The moving part is then drawn separately in more than one position representing its step-by-step movement on pieces of paper, and then the paper pieces are inserted to fit the empty space, so that the character moves and works in a particular environment without having to draw the character and the environment around it at a time, that is, the background is fixed and some of the character parts, and some of the other parts of the character are moving and separate .(Giannalberto Bendazzi, 2016, p39).

This can be seen in limited animation in social media, where the user moves simple parts of the character and installs the rest of the scene, so that the movement is mouth, eye or limb, and the number of frames per second is small, where the movement in the scene is sharp and clear

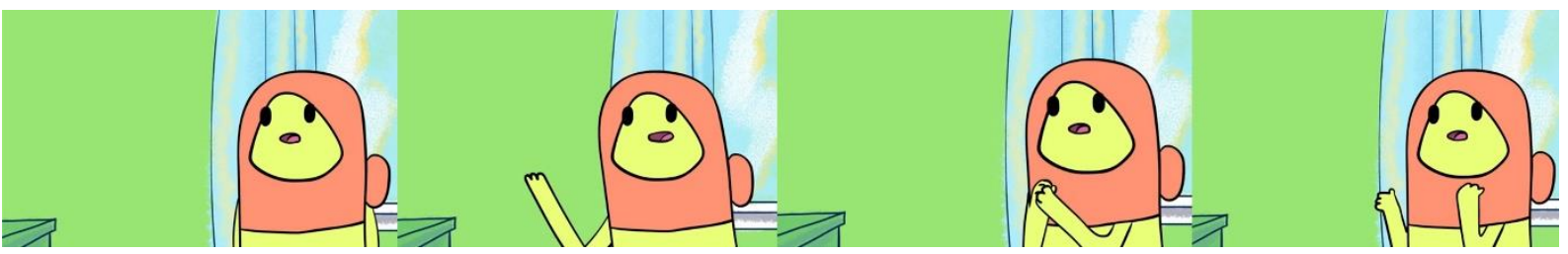

Fig.3. The slash system- Tahaleb - 2020

\subsubsection{Limited animation features in social media:}

Spontaneous aspect :The limited animation is similar in its spontaneity and nature to that created by ancient man for the innate expression of thought, with the expression used differing between cave walls and social media pages. Despite this difference in mediocrity and time, the similarity here comes in spreading individual human thought without paying more attention to aesthetic than expressionistic. 

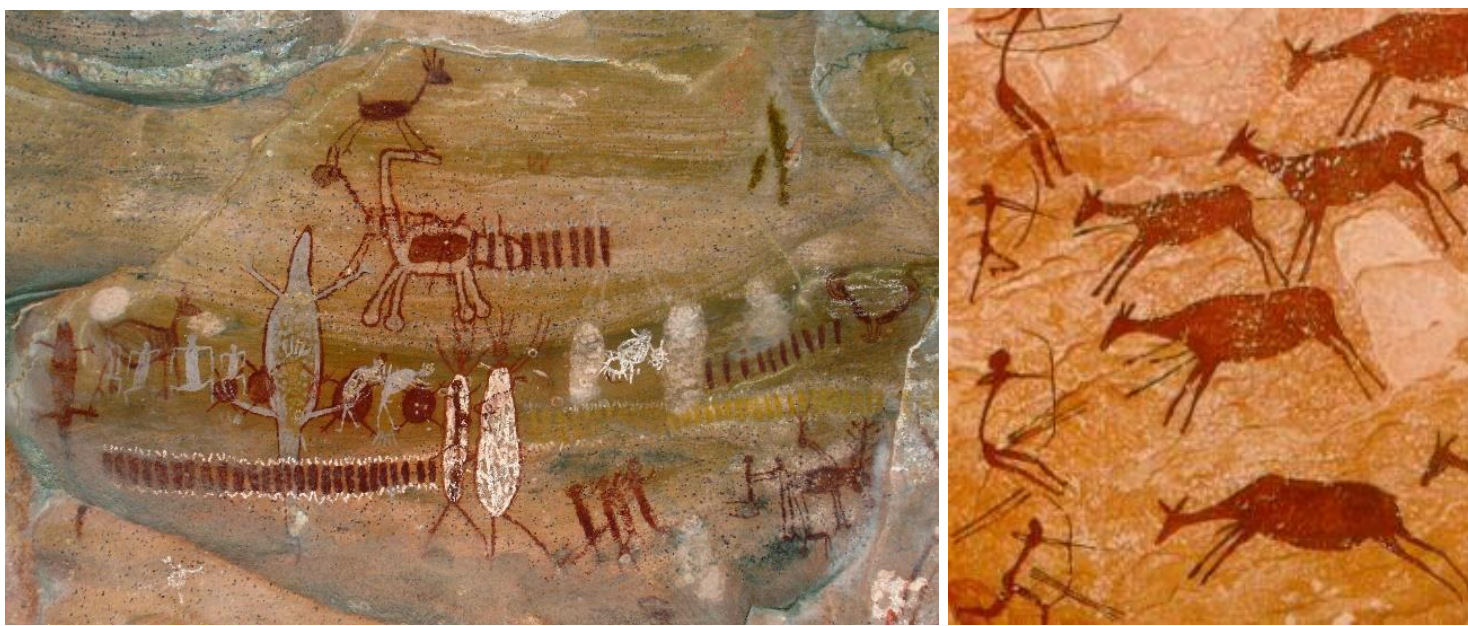

Fig.4. painting cave - Spontaneous aspect:

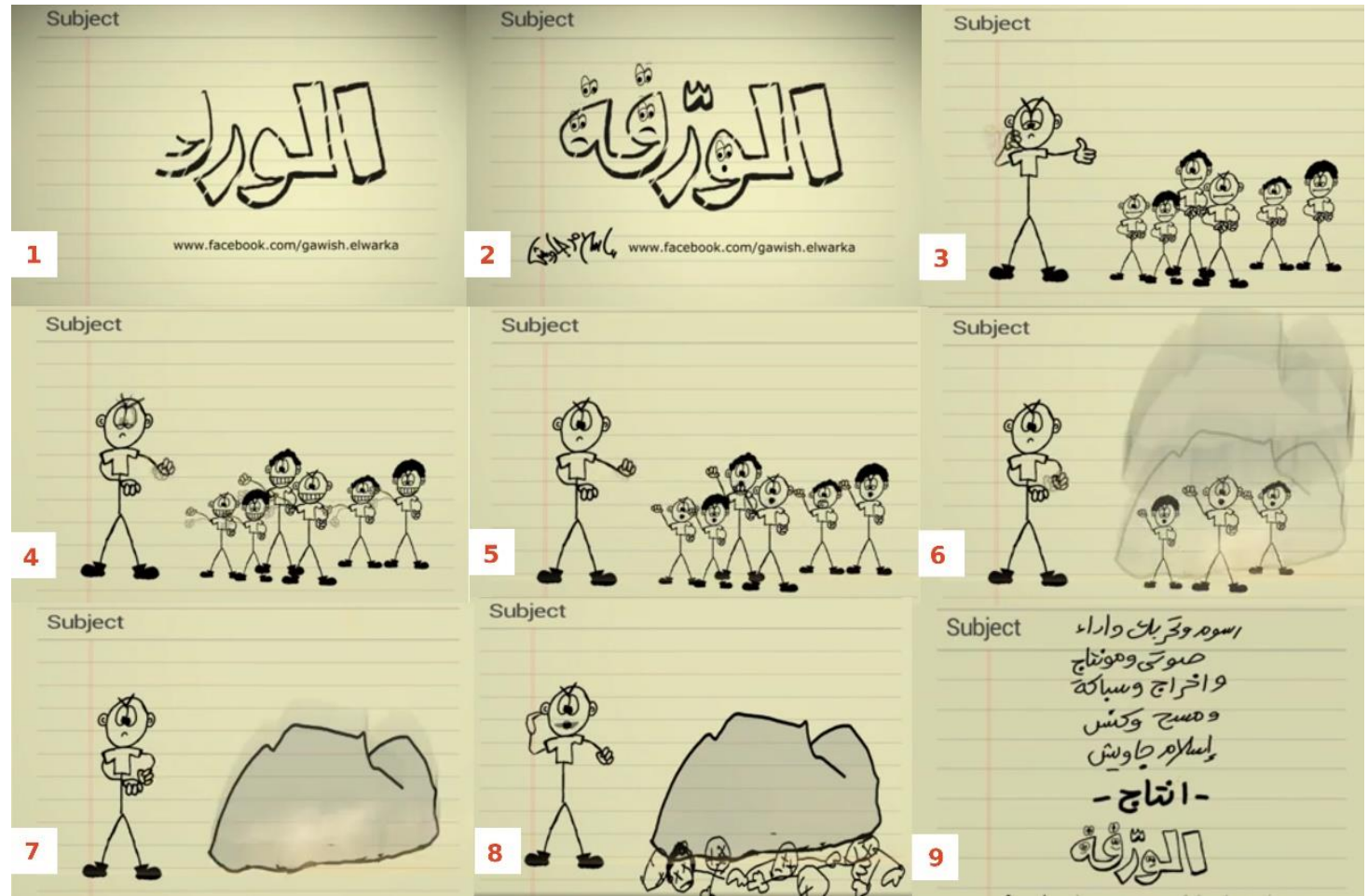

Fig.5. Spontaneous aspect in limited animation - Waraqa - Facebook - Eslam Gawish 2014

Abstract aspect :The abstract aspect of limited animation appears as a result of the speed of execution or the user's inexperience with the representative aspect of the graphics. The media content maker's interest in the delivery of intellectual content shows greater interest in the visual 
symmetry of animated elements, with some characters appearing stripped of the humanistic nature of reality.

Symbolic aspect :This aspect of finite animation is achieved by using the information organization of visual elements in so-called semantic coding .It refers to an iconic visual connotation, as seen in the expression of faces and body movement and the appearance of certain signs, and a formative connotation, as shown in the connotations of color and font movement, and semantic coding and representation of information are done in a specific system and under specific strategies such as:

- Serial Processing Strategy :The elements are treated visually one by one, where the recipient's attention is drawn to the object while the other object is overlooked, and the other object is moved sequentially, depending on its importance, as well as the strategy of selecting the object identified at the reception stage to focus on it and address it and not the other object. This is reflected in the highlight of some elements and the apparent omission of the background, until in some scenes it reaches a mere color.

- Parallel Processing Strategy :In these, a combination of effects is processed simultaneously independently of each other; That is, a set of mental processes are carried out on these stunts in isolation, and then during the processing stages they focus on each other and neglect each other, such as the elements being identified in black lines to give them greater attention, or having two elements moving in parallel in the same scene
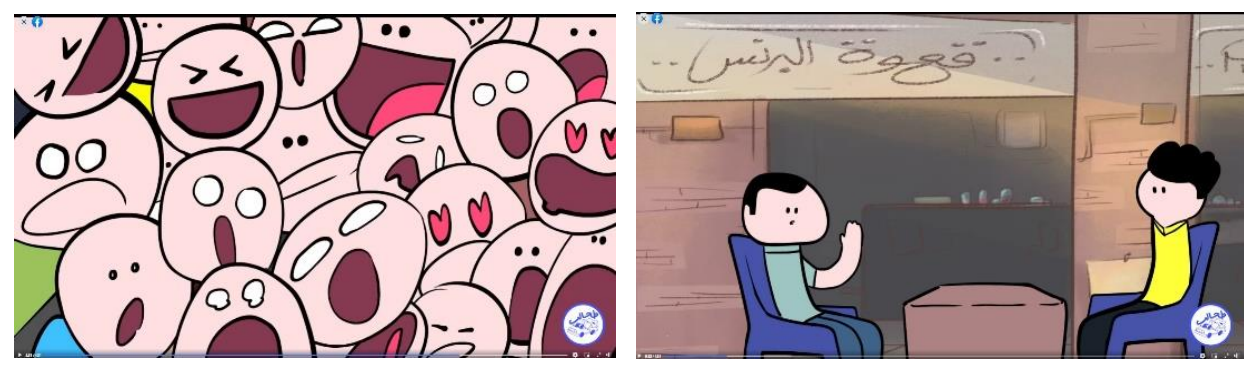

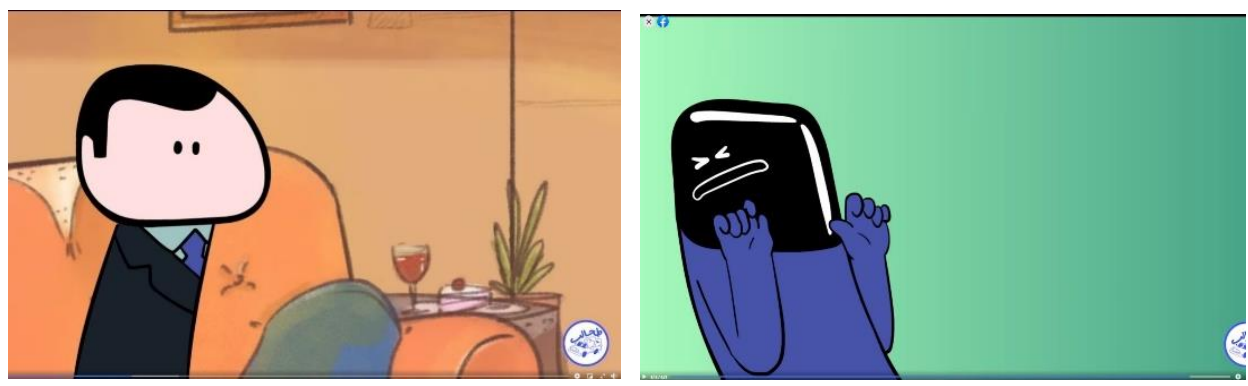

Fig.6. Symbolic aspect in limited animation - Tahaleb - Facebook - Episode 9 "Ahmed Alaadi"- 2020

\subsubsection{Relationship between limited animation features and the social media concept:}

The concept of social media is based on the principle of participation, speed, intensity and brevity. The content of the media is shared by individual volunteers with a view to disseminating their ideas without profit or remuneration, as in the traditional press, called "Participatory Culture," which brings together more than one individual, contributing to the formation of social capital (Soraya Ahmed El Badawi, 2015, p5).

In addition to the concept of participation, media contents are unique in a new way, as the individual can download his intellectual production and experiences at the instantaneous speed and the speed of circling the Internet, and the faster the spread and penetration, the stronger the circles of influence, which is somewhat similar with the theory of Cultivation theory. Or the development process, on which the concept of the recipient's watching of television was founded. The repetition of displaying some content to the viewer inculcates some cultures in the viewer involuntarily on his part and voluntarily from the state, to turn later into images and values that the viewer acquires about the real world (Hassan Emad Mekawi, Laila Husin Elsaid, 2010, p302).

As for social media sites, the faster the spread, the greater the opportunity for the user to communicate his or her mind more widely as a result of the increase in the audience, while if the user has less presence and fewer media content, then it can easily be skipped by the fact that it is not always present and the user's direction of other content. 
Aside from participation and speed of time, the intensity and the brevity of social media is such that writing on social media requires a condensation and a reduction of meaning. The technical template and the nature of the recipient of this type of writing do not allow him to indulge. The recipient here is not an experienced readership, and he has a long breath to follow up on literary results ' . Rather, he is newly interested in literature by the emergence of modern techniques, so the intensification of meaning is necessary to attract the attention of these broad categories of recipients (Basma Kqed Albanaa, 2014, P87).

From here comes the function of limited animation as a user's support, and in proportion to the concept of social media sites, limited animation is essentially controlled by the speed of time in its production, and thus shows the innate aspect, which is based on the spirit of the preparatory graphics that the user himself carries out, and the symbolic abstract aspects that depend on the infinite. It can therefore be argued that the link between limited animation and the concept of social media is the element of time with the speed of participation, condensation and brevity.

Full animation is attractive to the viewer and provides the aesthetic aspects, but sometimes it may not be with the high connectivity of limited animation to communicate the user's individual intellectual aspects clearly to the meaning and succinctly at once. The difference between full animation and limited animation is more like the difference between a long novel and a short story.

\subsection{Reasons for the appearance of limited animation in popular media from media theories:}

\subsubsection{Virtual identity theory and self-manipulation}

This principle is based on the fact that an individual in virtual identity often manipulates one's identity and takes other roles in order to induce others to interact with him/her. He/she may be a man and act as a woman, or be a student and act as a professor, so-called selfmanipulation, and that imaginary identity seeks to jump on the taboos of social identity.

Self-manipulation may come here not only to change true identity into a virtual identity, where virtual identities have emerged in the form of limited cartoon characters to address social media audiences with this identity, so that they can spread individual thought and beliefs through that personality, and for example, a user has created a channel of their own called. " 
Noor's World," which emerged as a virtual character calling for openness, is fighting another virtual character, a character. "Unjust," which calls for extremism. Through this channel, the user propagates his individual thinking through constant drawings and limited animation to raise public awareness of the dangers of what he sees as religious extremism, and to spread his thought calling for sound openness. Through this channel, he may be putting this extreme antipathy on the taboo of Arab social identity by mobilizing the public towards the extreme of another kind, namely, extreme openness.

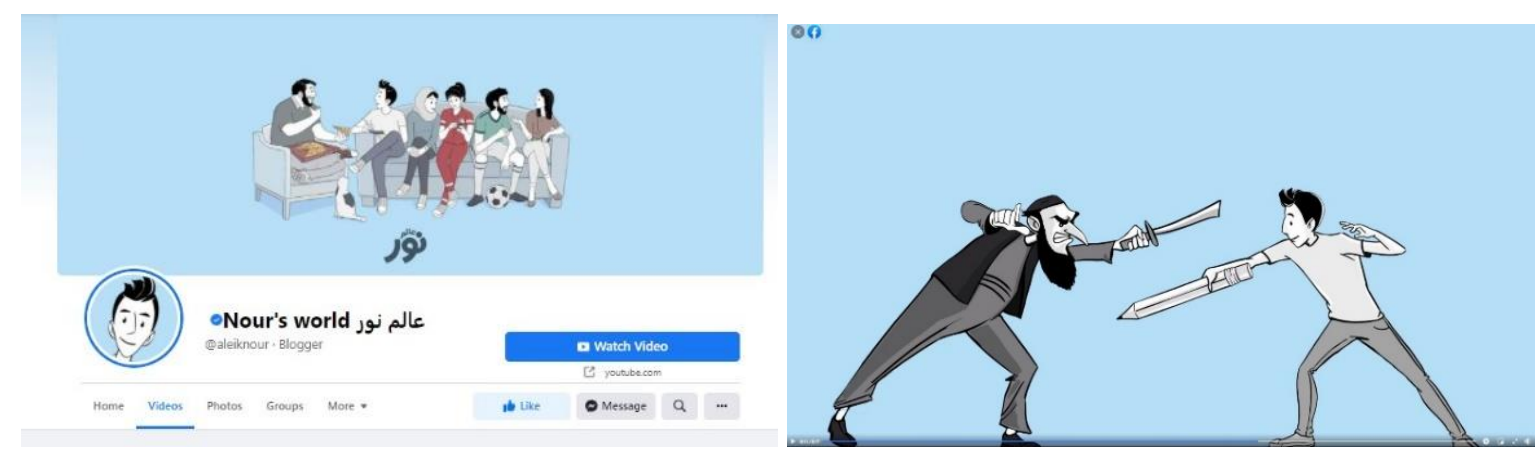

Fig.7. Virtual identity theory and self-manipulation- Nour's world - Facebook - 2017

\subsubsection{Dependency Theory:}

The basic premise of this theory is that interdependence plays an essential role in the exchange of information affecting personal and collective beliefs and decisions, benefits and even protests and strikes, bearing in mind that interdependence is shaped by the strength of the links between individuals and groups. The power of links to different motivations for the use of social networks varies ، Recreational motives differ from social motives, which in turn differ from political motives, while noting the dynamic of relationships across social networks, as they can be weakened because they are linked to the interests of individuals, who may be relaxed in strength and affect the effectiveness of interdependence (Mohamed Mostafa Refaat, 2018, P50).

From the reliance theory, the personal motives of individuals who use limited animation as a tool of expression through social media, or the personal motives of the individual receiving it, stem from their own spheres of influence, Limited animation depends on three aspects: 
- Cognitive Effect: through limited animation, the ambiguity resulting from the lack of sufficient information to understand an event, by providing adequate information and correct and close interpretations in the language of the simple recipient, limited animation, despite the simplicity of the movement, affects the audience's perception because of the relative importance it gives to some The issues it addresses for some issues, such as issues of values, beliefs and religion, and is done by simplifying the event and representing it visually and in motion.

- Affective Effect: it is the aspect associated with emotions, such as the increased tension and fears of the recipient about an event or its desire for something, achieved through the expression aspect of limited animation.

- Behavioral Effects: This aspect is the outcome of the cognitive effects of the communication of thought, of the emotional effects of the communication of emotion. When thought and feeling come together in media content, it shapes the intellectual and behavioral trends of the individual recipient (Basma Kqed Albanaa, 2014, p34, 35).

This was represented on the "Konooz" page, an educational advocacy channel on a website. Created to disseminate Islamic religion principles and transactions in the form of simplistic and attractive animation, Facebook and YouTube combine the cognitive influence aspect of Islamic religion and Islamic transactions, the emotional influence aspect of limited animation, and the behavioral impact aspect, where cognitive and affective aspects work together to revive Islamic religion teachings and how to deal with Islamic religion, and so there's a change in the behavior of society.
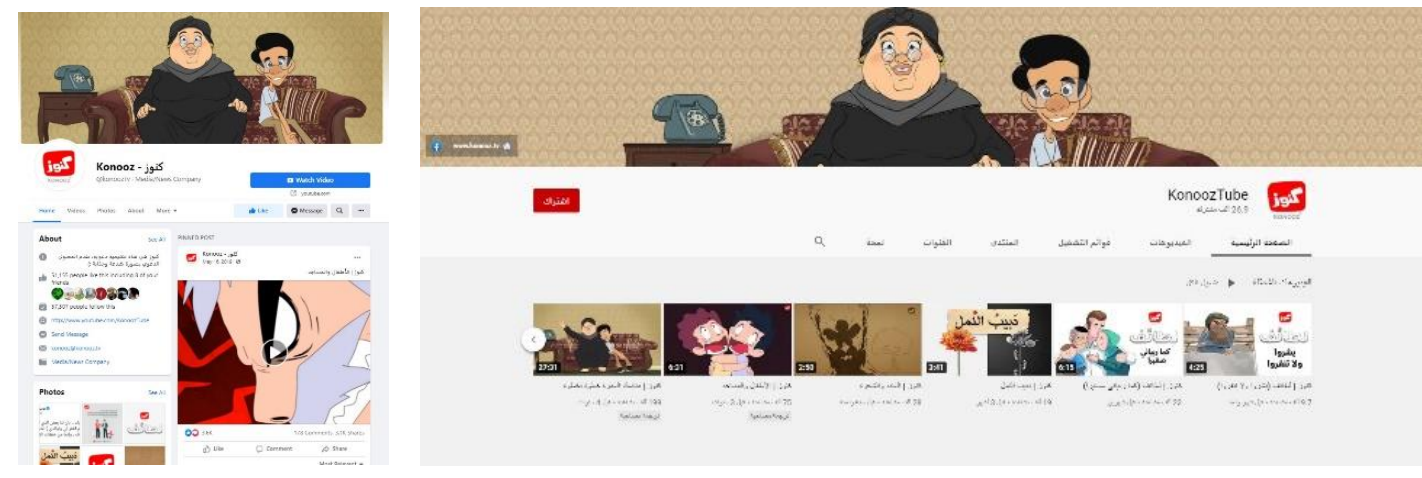

Fig.8. "Konooz" - Facebook and YouTube - 2018

\subsubsection{Theory of uses and satisfaction of needs:}


This theory assumes that the public is active and positive in interacting with the media with a view to satisfying their psychological and social needs. The public selects a particular media, or messages to satisfy a particular need, and by applying this theory to limited animation and its appearance on social media ‘.There are user-specific motives and rumors (content manufacturer - content recipient) to interact with or produce limited animated media content for others, divided into:

- beneficial motives, aimed at self-identification and the acquisition of knowledge, information and experience, and all forms of learning acquired.

- Ritual motives, aimed at spending time, venting, relaxing and spending free time.

- Social motives for free expression of opinion on different subjects and issues.

- Motives for using new media and controlling content.

- revelations of obtaining information, discovering reality, linking information obtained by an individual with their cultural inventory for the recipient, and the ability to communicate with others and form relationships for the media content maker (Basma Kqed Albanaa, 2014, p35, 36, 37).

\subsubsection{Theory of Symbolic Interactions}

And it's a theory that is concerned with the psychosocial dynamics of individuals interacting in social networks, and it focuses on concepts and meanings, which have been found and maintained through symbolic interaction between individuals. Our identity or sense of self is shaped by social interaction, and the concept of self is also shaped by how others interact with us, and it is among the appropriate theoretical frameworks for studying social existence in virtual society, Individuals interact through social networks through the use of text, audio and video, or digital characters, and these means represent their meaning and symbols. Users act in the virtual community through what things mean to them, and they form electronic selves by interacting with others.

\subsubsection{Theory of Media Richness:}

In its view, the effectiveness of communication depends on the extent to which it is used, and the theory focuses more on interactive forms of communication in two directions between the communicator and the future audience of the message. According to the media richness 
theory, the media that provides feedback is richer, the less ambiguous the more effective communication is.

The theory posits that the media have the ability to solve the ambiguity facing the public, provide diverse explanations and facilitate the process of understanding the future audience of the message. The richness of information is the process in which information reduces the degree of ambiguity, and creates a space of common meaning using a particular means of communication, which is achieved by limited animation towards highly effective communication of the knowledge aspect to the recipient, because of its visual and audio aspects.

\subsubsection{Convergence Theory:}

The content of the theory revolves around the fact that there are significant changes in the nature of human communication by new information technologies in terms of communication methods, which are meant to be the overlapping flow of different types of content - text, videos, images, as well as various audience activities - that have become capable of producing and selecting content within different social networks. One such activity is limited animation, which can be accompanied by sound, music and text so that it can be a powerful factor in attracting audiences to it (Mohamed Mostafa Refaat, 2018, p50, 59).

\section{Materials and methods:}

This research was based on the connection between different sciences with a view to achieving new research results. The limited animation emanating from the arts, the social sciences based on the study of virtual society, the movement of the individual to spread individual thought and mobilize and polarize the masses, and the psychology concerned with the recipient's perception of visual ideas.

- To this end, the research aimed to highlight limited animation as a modern phenomenon in electronic media, explain its existence from media theories, and clarify the characteristics of limited animation as an expression tool. The research assumed that limited animation has characteristics that are linked to the concept of social media and contribute effectively to the dissemination of individual ideas, the research therefore followed the descriptive and analytical approach to establishing this hypothesis and, to that end, three themes were presented: 
- Individual intellectual exchange in virtual societies

- Individual expression in popular media

- Reasons for the appearance of limited animation in popular media from media theories

\section{Results and discussion:}

- Limited animation plays a role in the dissemination of individual thought and the mobilization of public opinion. It is based on the concept of the challenge of silence through its dissemination in popular media, and has emerged as a medium of expression in individual media.

- There is a relationship between limited animation and the concept of social media, and the link between them is time, with its speed of participation, intensification and brevity.

- There are different techniques for limited animation that differ from full animation, and their existence affects the element of time, including:

- The Staccato system

- The Stretch system

- The slash system

There are several reasons why limited animation appears on social media, including:

- The tendency of individuals to express through a virtual identity to increase freedom of expression or to manipulate and jump on societal values and to spread an attractively contrary thought.

- The tendency of individuals to influence the audience cognitively, emotionally and behaviorally, which is achieved by limited animation.

- The need for individuals to express and publish their individual thought without censorship and their presence as an influential part in the virtual community, and the need for others to satisfy their cognitive aspects towards accessing simplified and concise information.

- Individuals need to interact with the virtual community through attractive media visual content.

- The richness of limited animation as a medium that can be used to create a media that is full of cognitive and emotional aspects. 
- Ease of selecting the animation by the recipient due to the fact that it contains a different texture of graphics, sound and sometimes texts.

\section{Conclusion:}

The research revolves around limited animation as a means of spreading individual thought through social networking sites. Social networking sites arose to represent virtual communities, and limited animation appeared as a means of individual intellectual exchange in these virtual communities, due to its freedom of expression and mobilization of virtual public opinion, defying silence. The emergence of popular media or citizen media has led to the fragmentation of the mass of the masses and the emergence of the so-called individual media.

As a result of the emergence of individual media in social networking sites, individuals resorted to using technology under the concept of rapid and concise participatory social networking sites, so limited animation appeared with its innate and abstract symbolic characteristics to suit this concept, and the user used different techniques to create limited animation such as the technique of animation through slicing, Or stirring through tension, or stirring through the movement of parts, and these techniques emerge from the movement of the few frames per second unit, causing the movement of sharp elements.

The emergence of limited animation had many reasons, and these reasons have been studied from the reality of media theories of popular media, such as the theory of virtual identity and self-manipulation, the theory of dependence, the theory of uses and satisfaction of needs, the theory of symbolic interaction, the theory of media richness, and the theory of convergence or integration.

The importance of this research comes by clarifying that there is a strong interrelationship between the human sciences, limited animation as a means of expression that emanates from the arts, has a close relationship with virtual communities that emerge from sociology, in addition to the techniques of animation movement and the distribution of elements within an animated work are science It stems from psychology, which studies the human visual perception and how to control it in order to communicate individual thought in an effective cognitive, emotional and behavioral way. 


\section{Reference:}

\section{Arabic References:}

1. Ahmed El Badawi, Soraya (2015). Mostakhdem alenternet: qtaa fi nazariat alealaam algedid wa manahego. T1. Egypt. Cairo: aalam alkotob.

2. Alaa Elde, Nermin (2020). Edaret estrategiat tawasol almonazamat abr wasael altawasol alegtemaai. Egypt, Cairo: alaraby lelnashr wa altawz.

3. Atef, Noha (2016). alealaam alshaabi: bin ealaam aldawla wa dawlet alealam. Eygpt, T1. Cairo: alaraby lelnashr wa altawz.

4. Emad Mekawi, Hassan, Husin Elsaid, Laila (2010). Aletesal wa nazariato almoasera. T9. Egypt. Cairo: aldar alebnania almasria.

5. Kqed Albanaa, Basma (2014). Tweeter wa albenaa alegtemaai wa althakafi lada alshabab. T1, Lebnan, Beirut: almassa alarabia lelderasat wa alnashr.

6. Mohamed Gazi, Khaled (2010). Alsahafa alelektronia alarabia, T1. Egypt, Algiza: wekalat alsahafa alarabia.

7. Refaat, Mohamed Mostafa (2018). Alrai alaam fe alwaqe aleftradi wa qwat al tabeaa aleftradia. T1. Egypt. Cairo: alaraby lelnashr wa altawz.

\section{Foreign References:}

1. Bashara, D (2019). Cartoon Vision: UPA Animation and Postwar Aesthetics. University of California Press. California. USA P5, p6

2. Bendazzi, Giannalberto (2016). Animation: A World History: Volume I: Foundations - The Golden Age. Focal press. p39

3. Frank, H (2019). Frame by Frame: A Materialist Aesthetics of Animated Cartoons. University of California Press. California. USA P75

4. Whitaker, H. Halas, J. Sito,T (2009). Timing for animation. Focal press. $2^{\text {nd }}$ edition. USA P1

\section{Website:}

1. https://www.dailymotion.com/video/x4j2nx4

2. https://www.facebook.com/eslam.hamed.545/videos/2822752547973900 
3. https://glennmagraphics.wordpress.com/tag/cave-art/

4. https://www.facebook.com/aleiknour/?ref=page_internal

5. https://www.facebook.com/konoozTv/?hc_ref=ARQhX8Q65hzhiGJGJCZ_8Vqz3diePBVbx9noIID_tk4B1Gmo7vDdDJ7Agvt9fS4Gtg\&fref=nf

6. https://www.youtube.com/user/KonoozTube

Received: April 2021

Accepted: June 2021 